\title{
Thermal QCD Sum Rules for $\sigma(600)$ Meson
}

\author{
Elşen Veli Veliev *, Takhmassib M. Aliev ** \\ * Physics Department, Kocaeli University, Umuttepe Yerleşkesi \\ 41380 Izmit, Turkey \\ e-mail: elsen@kocaeli.edu.tr \\ ** Physics Department, Middle East Technical University, \\ 06531 Ankara, Turkey \\ e-mail: taliev@metu.edu.tr
}

\begin{abstract}
In the present work, the temperature dependence of the scalar mesons parameters is investigated in the framework of thermal QCD sum rules. We calculate $\sigma$-pole and the non-resonant two-pion continuum contributions to the spectral density. Taking into account additional operators appearing at finite temperature, the thermal QCD sum rules are derived. The temperature dependence of the shifts in the mass and leptonic decay constant of scalar $\sigma(600)$ meson is calculated.
\end{abstract}

\section{Introduction}

The QCD sum rules method [1], proposed about three decades ago, is one of the powerful methods for investigating the properties of hadrons. This method has been extensively used as an efficient tool to study the masses, decay form factors and so on [2]. In recent years there has been increasing interest in the modification of hadronic properties at finite temperature in order to understand the results of the heavy ion collision experiments.

The QCD sum rules method is extended to the finite temperature in [3] and finite temperature sum rules have several new features. One of them is the interaction of the current with the particles of the medium. This effect requires modifying hadron spectral function. The other novel feature is the breakdown of Lorentz invariance by the choice of reference frame [4]- 6 . Due to the residual $\mathrm{O}(3)$ symmetry more operators with the same dimension appear in the operator product expansion (OPE) at finite temperature compared to those at zero temperature. Taking into account both complications, the investigation of OPE for thermal correlator of the two vector currents, and the thermal QCD sum rules for vector mesons have been realized in 
[7] and [8], respectively. Also, nuclear medium modifications of meson parameters are widely discussed in the literature [9]-[10].

In the present work, we investigate the properties of the scalar $\sigma$ meson in the framework of thermal QCD sum rules. The basic idea of thermal QCD sum rules is to get information about temperature dependence of hadron parameters, studying the same correlator, both at high temperature where the quark-gluon plasma is expected and at low temperature, where the hadronic phase is dominated. Note that the nature of light scalar mesons is still an open problem and is the subject of intensive and continuous theoretical [11] and experimental investigations [12. Can we get any new information about the nature of the scalar mesons from the thermal QCD analysis? Present work is addressed to the investigation of this problem.

The paper is organized as follows. In section 2 we derive the thermal QCD sum rules for scalar $\sigma(600)$ meson. In section 3 we present our numerical calculations. This section also contains discussion and our conclusion.

\section{Thermal QCD sum rules for scalar sigma mesons}

In this section we construct the thermal sum rules for scalar $\sigma(600)$ meson. For this purpose we consider the thermal average of correlation function

$$
T(q)=i \int d^{4} x e^{i q \cdot x}\langle T(J(x) J(0))\rangle,
$$

where $J(x)=\frac{1}{\sqrt{2}}(\bar{u} u+\bar{d} d)$ is the interpolating current with the $\sigma$ meson quantum numbers. The thermal average of any operator is determined by following expression

$$
\langle O\rangle=\operatorname{Tr} e^{-\beta H} O / \operatorname{Tr}^{-\beta H},
$$

where $H$ is the QCD Hamiltonian, and $\beta=1 / T$ stands for the inverse of the temperature $T$ and traces are carried out over any complete set of states.

The fundamental assumption of Wilson expansion is that the product of operators at different points can be expanded as the sum of local operators with momentum dependent coefficients in the form:

$$
T(q)=\sum_{n} C_{n}\left(q^{2}\right)\left\langle O_{n}\right\rangle,
$$

where $C_{n}\left(q^{2}\right)$ are called Wilson coefficients, and $O_{n}$ are a set of local operators. In this expansion, the operators are ordered according to their dimension $d$. The lowest dimension operator with $d=0$ is the unit operator associated with the perturbative contribution. In the vacuum sum rules operators with dimensions $d=3$ and $d=4$ composed of quark and gluon fields 
are the quark condensate $\langle\bar{\psi} \psi\rangle$ and the gluon condensate $\left\langle G_{\mu \nu}^{a} G^{a \mu \nu}\right\rangle$, respectively. At finite temperature Lorentz invariance is broken by the choice of a preferred frame of reference, and therefore new operators appear in the Wilson expansion. In order to restore Lorentz invariance in thermal field theory, four-vector velocity of the medium $u^{\mu}$ is introduced. Using four-vector velocity and quark/gluon fields, we can construct a new set of low dimension operators $\left\langle u \Theta^{f} u\right\rangle$ and $\left\langle u \Theta^{g} u\right\rangle$ with dimension $d=4$, where $\Theta_{\mu \nu}^{f}$ and $\Theta_{\mu \nu}^{g}$ are fermionic and gluonic parts of energy momentum tensor $\Theta_{\mu \nu}$, respectively. So, we can write thermal correlation function in terms of operators up to dimension four:

$$
T(q)=C_{1} I+C_{2}\langle\bar{\psi} \psi\rangle+C_{3}\left\langle G_{\mu \nu}^{a} G^{a \mu \nu}\right\rangle+C_{4}\left\langle u \Theta^{f} u\right\rangle+C_{5}\left\langle u \Theta^{g} u\right\rangle
$$

The Wilson coefficients in Eq.(44) are calculated in [13], and renormalization group improved expression of OPE leads to the following result

$$
\begin{aligned}
& T(Q)=\frac{3}{8 \pi^{2}} Q^{2}\left(\gamma-\ln \frac{4 \pi}{Q^{2}}\right)+\frac{3}{Q^{2}} m\langle\bar{\psi} \psi\rangle+\frac{g^{2}}{32 \pi^{2} Q^{2}}\left\langle G_{\mu \nu}^{a} G^{a \mu \nu}\right\rangle \\
& +\frac{4}{16+3 n_{f}}\left(\frac{4(u \cdot Q)^{2}}{Q^{4}}+\frac{1}{Q^{2}}\right)\left[\langle u \Theta u\rangle+\lambda\left(Q^{2}\right)\left(\frac{16}{3}\left\langle u \Theta^{f} u\right\rangle-\left\langle u \Theta^{g} u\right\rangle\right)\right]
\end{aligned}
$$

where $Q$ is the Euclidean momentum, $\lambda\left(Q^{2}\right)=\left[\alpha_{s}\left(\mu^{2}\right) / \alpha_{s}\left(Q^{2}\right)\right]^{-\delta / b}$ and $\Theta_{\mu \nu}=n_{f} \Theta_{\mu \nu}^{f}+\Theta_{\mu \nu}^{g}$. At one loop level the constants $\delta$ and $b$ are given by

$$
\delta=\frac{2}{3}\left(\frac{16}{3}+n_{f}\right) \quad \text { and } \quad b=11-\frac{2}{3} n_{f},
$$

where $n_{f}$ is quark flavours number. The spectral representation for the correlation function in $q_{0}$ at fixed $|\mathbf{q}|$ can be written as [8]

$$
T\left(q_{0}^{2},|\mathbf{q}|\right)=\int_{0}^{\infty} d q_{0}^{\prime 2} \frac{N\left(q_{0}^{\prime},|\mathbf{q}|\right)}{{q^{\prime}}_{0}^{2}+Q_{0}^{2}}+\text { subtraction terms }
$$

where

$$
N\left(q_{0},|\mathbf{q}|\right)=\frac{1}{\pi} \operatorname{ImT}\left(q_{0},|\mathbf{q}|\right) \tanh \left(\beta q_{0} / 2\right) \quad \text { and } \quad Q_{0}^{2}=-q_{0}^{2}
$$

Note that, the subtraction terms are removed by the Borel transformation. For this reason in further discussion we omit these terms. Equating spectral representation and OPE, and performing Borel transformations with respect to $Q_{0}^{2}$ from both sides Eq.(7), we obtain the QCD sum rules

$$
\int_{0}^{\infty} d q_{0}^{2} e^{-q_{0}^{2} / M^{2}} N\left(q_{0},|\mathbf{q}|\right)=e^{-|\mathbf{q}|^{2} / M^{2}}\left[\frac{3 M^{4}}{8 \pi^{2}}+\left\langle O_{1}\right\rangle+\left(1-\frac{4 \mathbf{q}^{2}}{3 M^{2}}\right)\left\langle O_{2}\right\rangle\right]
$$


where $M$ is Borel parameter, $\left\langle O_{1}\right\rangle$ and $\left\langle O_{2}\right\rangle$ are the non-perturbative contributions of higher dimensional operators,

$$
\begin{gathered}
\left\langle O_{1}\right\rangle=3 m\langle\bar{\psi} \psi\rangle+\frac{g^{2}}{32 \pi^{2}}\left\langle G_{\mu \nu}^{a} G^{a \mu \nu}\right\rangle, \\
\left\langle O_{2}\right\rangle=-\frac{12}{16+3 n_{f}}\left[\langle u \Theta u\rangle+\lambda\left(M^{2}\right)\left(\frac{16}{3}\left\langle u \Theta^{f} u\right\rangle-\left\langle u \Theta^{g} u\right\rangle\right)\right] .
\end{gathered}
$$

Now we consider the phenomenological part of the correlation function. We shall work below the critical temperature, where the physical spectrum is saturated by hadrons . In this case, similar to the vacuum QCD sum rules, the dominant contribution to the spectral density comes from $\sigma$ mesons. We also calculate the contribution of the non-resonant two-pion continuum.

Let us calculate $\sigma$-pole contribution to the correlator. The leptonic decay constant $\lambda_{\sigma}$ of the $\sigma$-meson is given by, $\langle 0|J(0)| \sigma\rangle=m_{\sigma} \lambda_{\sigma}$, where $m_{\sigma}$ is $\sigma$-meson mass. In thermal field theory, the parameters $m_{\sigma}$ and $\lambda_{\sigma}$ must be replaced by their temperature dependent values. The vacuum value of the leptonic decay constant is obtained from two point QCD sum rules and $\lambda_{\sigma}=200 \mathrm{MeV}$ [14]. The absorptive part of the correlation function is calculated by using the following field-current identity

$$
J(x)=m_{\sigma} \lambda_{\sigma} \sigma(x),
$$

and $\sigma$-meson contribution to thermal correlator can be written as

$$
T(q)=i m_{\sigma}^{2} \lambda_{\sigma}^{2} D_{11}^{\sigma}(q)
$$

Here $D_{11}^{\sigma}(q)=\int d^{4} x e^{i q \cdot x}\langle T(\sigma(x) \sigma(0))\rangle$ is the time ordered product of two $\sigma$-meson fields (11component of the finite temperature scalar field propagator with mass $m_{\sigma}$ in the real time formalism) and has the following form [15]-[16]

$$
D_{11}^{\sigma}(q)=\frac{i}{q^{2}-m_{\sigma}^{2}+i \varepsilon}+2 \pi n\left(\omega_{q}\right) \delta\left(q^{2}-m_{\sigma}^{2}\right)
$$

where $n\left(\omega_{q}\right)$ is the Bose distribution function, $n\left(\omega_{q}\right)=\left[\exp \left(\beta \omega_{q}\right)-1\right]^{-1}$ and $\omega_{q}=\sqrt{\mathbf{q}^{2}+m_{\sigma}^{2}}$. The imaginary part of correlation function can be simply evaluated using the formula $\frac{i}{x+i \varepsilon}=$ $\pi \delta(x)+i P\left(\frac{1}{x}\right)$, which leads to

$$
\operatorname{ImT}(q)=\pi m_{\sigma}^{2} \lambda_{\sigma}^{2}\left(2 n\left(\omega_{q}\right)+1\right) \delta\left(q^{2}-m_{\sigma}^{2}\right)
$$

With the help of $\delta$-function we obtain the following result for $\sigma$-pole contribution to the spectral function

$$
N(q)=m_{\sigma}^{2} \lambda_{\sigma}^{2} \delta\left(q^{2}-m_{\sigma}^{2}\right)
$$


In order to calculate the dependence of $m_{\sigma}$ and $\lambda_{\sigma}$ on temperature, we consider appropriate loop diagrams. Let us calculate the $\pi \pi$-contribution to the amplitudes, which describes the interaction of the current with the particles in the medium. This contribution to the correlation function can be written as

$$
T(q)=i g_{\sigma}^{2} \int \frac{d^{4} k}{(2 \pi)^{4}} D_{11}^{\pi}(k) D_{11}^{\pi}(k-q),
$$

where $D_{11}^{\pi}(k)$ is the 11-component of the finite temperature propagator for pions and $g_{\sigma}=$ 2, $0 \mathrm{GeV}$ [17]-[18]. The integration over $k_{0}$ in Eq.(17) can be evaluated using the residue theorem. After integration and some simplifications for the imaginary part of the correlation function we obtain

$$
\begin{aligned}
\operatorname{ImT}(q) & =\pi g_{\sigma}^{2} \int \frac{d \mathbf{k}}{(2 \pi)^{3}} \frac{1}{4 \omega_{1} \omega_{2}}\left(\left(1+n_{1}\right)\left(1+n_{2}\right)+n_{1} n_{2}\right)\left(\delta\left(q_{0}-\omega_{1}-\omega_{2}\right)+\delta\left(q_{0}+\omega_{1}+\omega_{2}\right)\right) \\
& +\left(\left(1+n_{1}\right) n_{2}+\left(1+n_{2}\right) n_{1}\right)\left(\delta\left(q_{0}-\omega_{1}+\omega_{2}\right)+\delta\left(q_{0}+\omega_{1}-\omega_{2}\right)\right)
\end{aligned}
$$

where,

$$
n_{1}=n\left(\omega_{1}\right), \quad n_{2}=n\left(\omega_{2}\right), \quad \omega_{1}=\sqrt{\mathbf{k}^{2}+m_{\pi}^{2}}, \quad \omega_{2}=\sqrt{(\mathbf{k}-\mathbf{q})^{2}+m_{\pi}^{2}} .
$$

At values $q_{0}=\omega_{1}+\omega_{2}$ and $q_{0}=\omega_{1}-\omega_{2}$ the terms involving the density distributions can be written as

$$
\begin{gathered}
{\left[\left(1+n_{1}\right)\left(1+n_{2}\right)+n_{1} n_{2}\right] \tanh \left(\frac{\beta q_{0}}{2}\right)=\left(n_{1}+n_{2}+1\right),} \\
{\left[\left(1+n_{1}\right) n_{2}+\left(1+n_{2}\right) n_{1}\right] \tanh \left(\frac{\beta q_{0}}{2}\right)=\left(n_{2}-n_{1}\right),}
\end{gathered}
$$

respectively. As can be seen, delta function $\delta\left(q_{0}-\omega_{1}-\omega_{2}\right)$ in Eq.(18) gives the first branch cut, $q^{2} \geq 4 m_{\pi}^{2}$, which coincides with zero temperature cut that describes the standard threshold for particle decays. On the other hand, delta function $\delta\left(q_{0}-\omega_{1}+\omega_{2}\right)$ in Eq.(18) shows that an additional branch cut arises at finite temperature, $q^{2} \leq 0$, which corresponds to particle absorption from the medium. Therefore, delta functions $\delta\left(q_{0}-\omega_{1}-\omega_{2}\right)$ and $\delta\left(q_{0}-\omega_{1}+\omega_{2}\right)$ in Eq.(18) contribute in regions $q^{2} \geq 4 m_{\pi}^{2}$ and $q^{2} \leq 0$, respectively. Taking into account both contributions, the spectral function can be written as

$$
\begin{aligned}
N(q)= & g_{\sigma}^{2} \int \frac{k^{2} \sin \theta d k d \theta}{(2 \pi)^{2} 2 \omega_{1}}\left[\left(n_{1}+n_{2}+1\right) \theta\left(q^{2}-4 m_{\pi}^{2}\right)+\left(n_{2}-n_{1}\right) \theta\left(-q^{2}\right)\right] \\
& \times \delta\left(q^{2}-2 q_{0} \omega_{1}+2|\mathbf{k}||\mathbf{q}| \cos \theta\right) .
\end{aligned}
$$

The integration over angle $\theta$ in Eq.(22) can be evaluated using the constraint $\left|\cos \theta_{\mathbf{q}, \mathbf{k}}\right| \leq 1$, which leads to following inequality

$$
\frac{\left|q^{2}-2 q_{0} \omega_{1}\right|}{2|\mathbf{k}||\mathbf{q}|} \leq 1
$$


The solution of this inequality at values $q^{2} \geq 4 m_{\pi}^{2}$ give us the integration range of $\omega_{1}$ as $\omega_{-} \leq \omega \leq \omega_{+}$, where

$$
\begin{gathered}
\omega_{ \pm}=\frac{1}{2}\left(q_{0} \pm|\mathbf{q}| v\right) \\
v\left(q^{2}\right)=\sqrt{1-4 m_{\pi}^{2} / q^{2}}
\end{gathered}
$$

At $q^{2} \leq 0$ the region of variation of $\omega_{1}$ must be $\omega_{+} \leq \omega_{1}<\infty$. Finally, the thermal spectral function can be written as

$$
N(q)=\frac{g_{\sigma}^{2}}{2|\mathbf{q}|} \int_{\omega_{-}}^{\omega_{+}} \frac{d \omega_{1}}{(2 \pi)^{2}}\left(n_{1}+n_{2}+1\right) \theta\left(q^{2}-4 m_{\pi}^{2}\right)+\frac{g_{\sigma}^{2}}{2|\mathbf{q}|} \int_{\omega_{+}}^{\infty} \frac{d \omega_{1}}{(2 \pi)^{2}}\left(n_{2}-n_{1}\right) \theta\left(-q^{2}\right) .
$$

Changing the variable $\omega_{1}$ to $x$ given by $\omega_{1}=\frac{1}{2}\left(q_{0}+|\mathbf{q}| x\right)$, we finally get the two pion contribution to the spectral function as

$$
\begin{aligned}
& N(q) \equiv g_{\sigma}^{2} \frac{v\left(q^{2}\right)}{8 \pi^{2}}+N_{1}(q)=\frac{g_{\sigma}^{2} v\left(q^{2}\right)}{8 \pi^{2}}+\frac{g_{\sigma}^{2}}{8 \pi^{2}} \int_{-v}^{v} d x n\left(\frac{1}{2}\left(q_{0}+|\mathbf{q}| x\right)\right), \quad q^{2} \geq 4 m^{2} \\
& N(q) \equiv N_{2}(q)=\frac{g_{\sigma}^{2}}{16 \pi^{2}} \int_{v}^{\infty} d x\left[n\left(\frac{1}{2}\left(|\mathbf{q}| x-q_{0}\right)\right)-n\left(\frac{1}{2}\left(|\mathbf{q}| x+q_{0}\right)\right)\right], \quad q^{2} \leq 0
\end{aligned}
$$

The QCD sum rules are obtained by equating theoretical and phenomenological parts of correlation function. Taking into account of expressions Eq.(27) and Eq.(28) in Eq.(9) we get

$$
\begin{aligned}
& m_{\sigma}^{2}(T) \lambda_{\sigma}^{2}(T) e^{-m_{\sigma}^{2}(T) / M^{2}}+\frac{g_{\sigma}^{2}}{8 \pi^{2}} e^{|\mathbf{q}|^{2} / M^{2}} \int_{4 m_{\pi}^{2}+|\mathbf{q}|^{2}}^{\infty} d q_{0}^{2} e^{-q_{0}^{2} / M^{2}} v\left(q^{2}\right) \\
+ & e^{|\mathbf{q}|^{2} / M^{2}}\left(\int_{4 m_{\pi}^{2}+\left.\mathbf{q}\right|^{2}}^{\infty} d q_{0}^{2} e^{-q_{0}^{2} / M^{2}} N_{1}\left(q_{0},|\mathbf{q}|\right)+\int_{0}^{|\mathbf{q}|^{2}} d q_{0}^{2} e^{-q_{0}^{2} / M^{2}} N_{2}\left(q_{0},|\mathbf{q}|\right)\right) \\
= & \frac{3 M^{4}}{8 \pi^{2}}+\left\langle O_{1}\right\rangle+\left(1-\frac{4 \mathbf{q}^{2}}{3 M^{2}}\right)\left\langle O_{2}\right\rangle .
\end{aligned}
$$

As the temperature approaches to zero, the two terms in bracket go to zero and the thermal average of the operators on the right become the expectation values, recovering the vacuum sum rules [14]. In the limit $|\mathbf{q}| \rightarrow 0$, the sum rule (29) simplifies considerably. Finally we obtain that

$$
m_{\sigma}^{2}(T) \lambda_{\sigma}^{2}(T) \exp \left(-m_{\sigma}^{2}(T) / M^{2}\right)+I_{0}\left(M^{2}\right)+I_{1}\left(M^{2}\right)=\frac{3 M^{4}}{8 \pi^{2}}+\langle O\rangle
$$

where

$$
\begin{gathered}
I_{0}\left(M^{2}\right)=\frac{g_{\sigma}^{2}}{8 \pi^{2}} \int_{4 m_{\pi}^{2}}^{\infty} d s v(s) \exp \left(-s / M^{2}\right) \\
I_{1}\left(M^{2}\right)=\frac{g_{\sigma}^{2}}{4 \pi^{2}} \int_{4 m_{\pi}^{2}}^{\infty} d s v(s) n(\sqrt{s} / 2) \exp \left(-s / M^{2}\right) \\
\langle O\rangle=\left\langle O_{1}\right\rangle+\left\langle O_{2}\right\rangle
\end{gathered}
$$




\section{Numerical analysis of the shifts in mass and leptonic decay constant}

In this section we present our results for the temperature dependence of the shifts in $\sigma$ meson mass and leptonic decay constant. By derivativing with respect to $1 / M^{2}$ from both sides of the sum rules (30), and making some transformations we obtain

$$
\begin{gathered}
m_{\sigma}^{2}(T)=\frac{m_{\sigma}^{4} \lambda_{\sigma}^{2} \exp \left(-m_{\sigma}^{2} / M^{2}\right)-J_{1}\left(M^{2}\right)+\eta \overline{\left\langle O_{3}\right\rangle}}{m_{\sigma}^{2} \lambda_{\sigma}^{2} \exp \left(-m_{\sigma}^{2} / M^{2}\right)-I_{1}\left(M^{2}\right)+\overline{\langle O\rangle}}, \\
\lambda_{\sigma}^{2}(T)=\lambda_{\sigma}^{2} \frac{m_{\sigma}^{2} \lambda_{\sigma}^{2}+\left(\overline{\langle O\rangle}-I_{1}\left(M^{2}\right)\right) \exp \left(m_{\sigma}^{2} / M^{2}\right)}{m_{\sigma}^{2} \lambda_{\sigma}^{2}+\left(\frac{1}{M^{2}}-\frac{1}{m_{\sigma}^{2}}\right)\left[J_{1}\left(M^{2}\right)-\eta \overline{\left\langle O_{3}\right\rangle}+m_{\sigma}^{2}\left(\overline{\langle O\rangle}-I_{1}\left(M^{2}\right)\right)\right] \exp \left(m_{\sigma}^{2} / M^{2}\right)},
\end{gathered}
$$

where the bar on the operators means subtractions of their vacuum expectation values and

$$
\begin{gathered}
\eta\left(M^{2}\right)=\frac{\delta M^{2}}{b \ln \left(M^{2} / \Lambda^{2}\right)}, \\
J_{1}\left(M^{2}\right)=\frac{g_{\sigma}^{2}}{4 \pi^{2}} \int_{4 m_{\pi}^{2}}^{\infty} d s s v(s) n(\sqrt{s} / 2) \exp \left(-s / M^{2}\right), \\
\overline{\left\langle O_{3}\right\rangle}=-\frac{12}{16+3 n_{f}} \lambda\left(M^{2}\right)\left(\frac{16}{3}\left\langle u \Theta^{f} u\right\rangle-\left\langle u \Theta^{g} u\right\rangle\right) .
\end{gathered}
$$

For the numerical analysis, let us list thermal average of operators contributing to the QCD sum rules. The temperature dependence of quark condensate is known from chiral perturbation theory [19]-20]

$$
\langle\bar{\psi} \psi\rangle=\langle 0|\bar{\psi} \psi| 0\rangle\left[1-\frac{n_{f}^{2}-1}{n_{f}} \frac{T^{2}}{12 F^{2}}+O\left(T^{4}\right)\right],
$$

where $n_{f}$ is number of quark flavors and $F=0.088 \mathrm{GeV}$. The low temperature expansion of the gluon condensate has been studied in article [21]

$$
\frac{g^{2}}{4 \pi^{2}} \overline{\left\langle G_{\mu \nu}^{a} G^{a \mu \nu}\right\rangle}=-\frac{8}{9}\left(\left\langle\Theta_{\mu}^{\mu}\right\rangle+\sum_{f} m_{f} \overline{\langle\bar{\psi} \psi\rangle}\right),
$$

where the trace of the total energy momentum tensor $\Theta_{\mu}^{\mu}$ is given by $\left\langle\Theta_{\mu}^{\mu}\right\rangle=\langle\Theta\rangle-3 p$, and for two massless quarks in the low temperature chiral perturbation limit the trace has following form 19

$$
\left\langle\Theta_{\mu}^{\mu}\right\rangle=\frac{\pi^{2}}{270} \frac{T^{8}}{F_{\pi}^{4}} \ln \frac{\Lambda_{p}}{T}+O\left(T^{10}\right) .
$$

Here $\langle\Theta\rangle$ is the total energy density and $p$ is the pressure, whose expressions are known in the low temperature region [20]. The pion decay constant has the value of $F_{\pi}=0.093 G e V$ and the logarithmic scale factor is $\Lambda_{p}=0.275 \mathrm{GeV}$. We also use the fact that, the quark and 
gluon energy densities at finite temperature can be expressed as $n_{f}\left\langle\Theta^{f}\right\rangle=\left\langle\Theta^{g}\right\rangle=\frac{1}{2}\langle\Theta\rangle$, which agrees both with the naive counting of the degrees of freedom and empirical studies of the pion structure functions [4], 8].

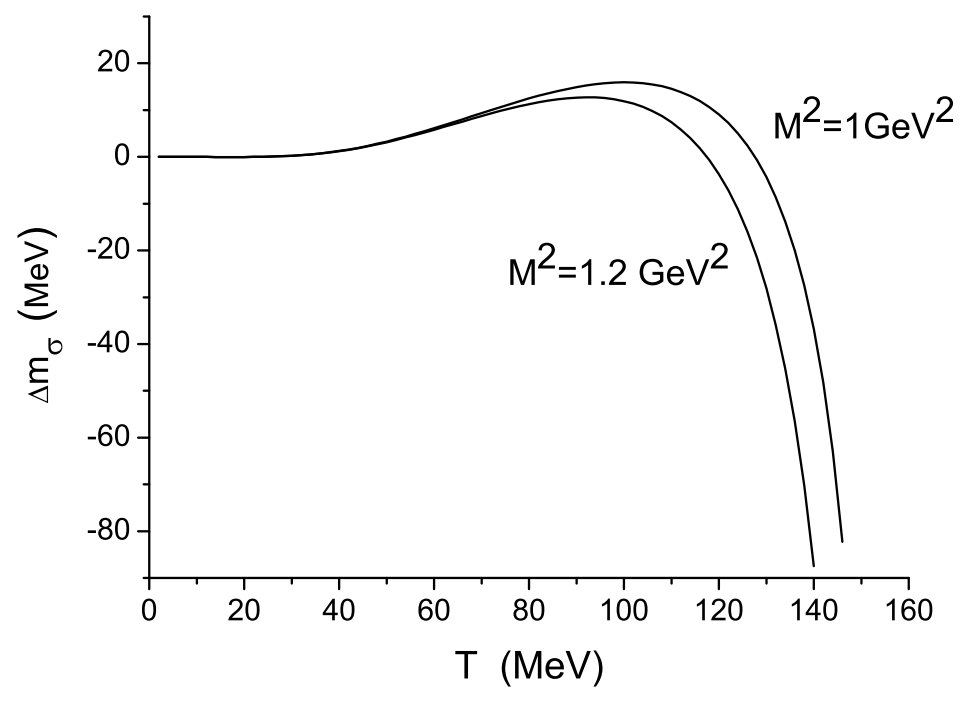

Figure 1: Shift in the sigma meson mass as a function of temperature for $M^{2}=1 \mathrm{GeV}^{2}$ and $M^{2}=1.2 \mathrm{GeV}^{2}$.

For the numerical evolution of the above sum rule, we use the values $m\langle\bar{\psi} \psi\rangle=-0.82 \times$ $10^{-4} \mathrm{GeV}^{4}, \Lambda=0.230 \mathrm{GeV}$ and $m_{\sigma}=0.6 \mathrm{GeV}$. We study the dependence of $\sigma$ meson mass and leptonic decay constant on $M^{2}$, when $M^{2}$ changes between $0.9 \mathrm{GeV}^{2}$ and $1.4 \mathrm{GeV}^{2}$. This region of $M^{2}$ is obtained from the mass sum rule analysis of the $\sigma$ meson [14].

The shifts in $\sigma$ meson mass and leptonic decay constant as a function of temperature for different values of $M^{2}$ is shown in Fig.1 and Fig.2, respectively. As seen, the results for $\Delta m_{\sigma}$ are stable for temperatures up to $120 \mathrm{MeV}$. At high temperatures the results for $\Delta m_{\sigma}$ becomes unstable and the contributions of higher dimensional operators become important here, whose inclusions might restore the stability in $M^{2}$ to higher temperatures. The results for $\Delta \lambda_{\sigma}$ are stable and leptonic decay constant decreases with increasing temperature and vanishes approximately at temperature $T=160 \mathrm{MeV}$. This situation may be interpreted as a signal for deconfinement and agrees with heavy-light mesons investigations [23]. Numerical analysis shows that the temperature dependence of $\Delta \lambda_{\sigma}$ is the same, when $M^{2}$ changes between $0.9 \mathrm{GeV}^{2}$ and $1.4 \mathrm{GeV}^{2}$.

Obtained results can be used for interpretation heavy ion collision experiments. It is also essential to compare these results with other model calculations. We believe these studies to 


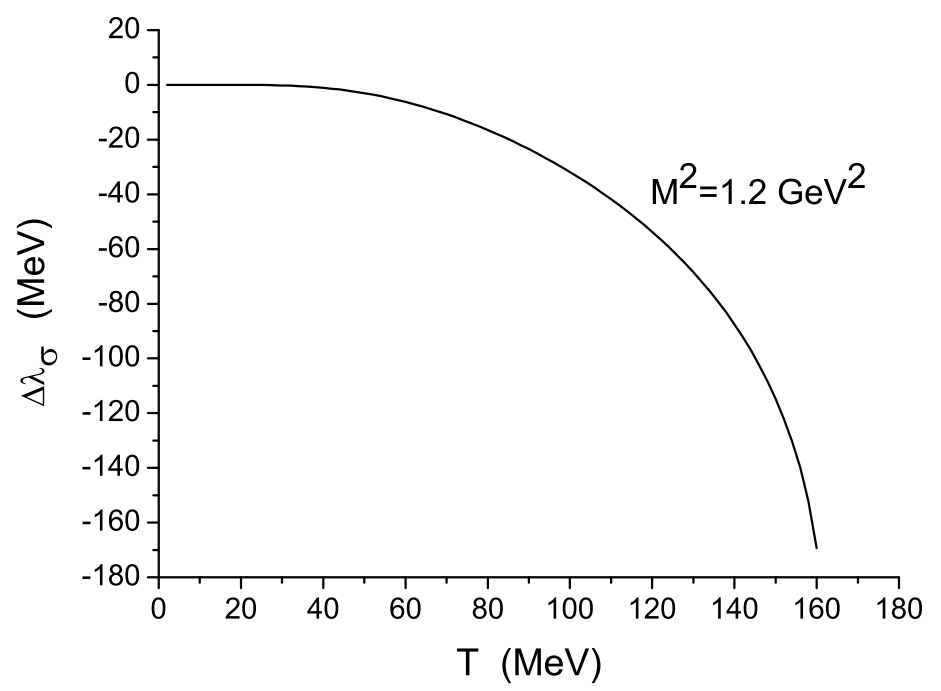

Figure 2: Shift in the leptonic decay constant as a function of temperature for $M^{2}=1.2 \mathrm{GeV}^{2}$. be of great importance for understanding phenomenological and theoretical aspects of thermal QCD.

\section{Acknowledgement}

This work is supported by the Scientific and Technological Research Council of Turkey (TUBITAK), research project no.105T131, and the Research Fund of Kocaeli University under grant no. $2004 / 4$.

\section{References}

[1] M.A. Shifman, A.I. Vainstein and V.I. Zakharov, Nucl. Phys. B 147, 385; M.A. Shifman, A.I. Vainstein and V.I. Zakharov, Nucl. Phys. B 147, 448 (1979).

[2] P. Colangelo, A. Khodjamirian, In: At the Frontier of Particle Physics, vol.3, ed. M. Shifman, World Scientific, Singapore, 1495 (2001).

[3] A. I. Bochkarev and M. E. Shaposhnikov, Nucl. Phys. B 268, 220 (1986).

[4] E.V. Shuryak, Rev. Mod. Phys., 65, 1 (1993).

[5] T. Hatsuda, Y. Koike, S. H. Lee, Nucl. Phys. B 394, 221 (1993). 
[6] T. Hatsuda, Y. Koike, S. H. Lee, Phys. Rev. D 47, 1225 (1993).

[7] S. Mallik Phys. Lett. B 416, 373 (1998).

[8] S. Mallik and K. Mukherjee, Phys. Rev. D58, 096011 (1998); S. Mallik and K. Mukherjee, Phys. Rev. D 61, 116007 (2000).

[9] V. L. Eletsky and B. L. Ioffe, Phys. Rev. Lett. 78, 1010 (1997).

[10] S. Mallik and A. Nyffeler, Phys. Rev. C 63, 065204 (2001).

[11] N.A.Tornqvist, Invited talk at conference: "Possible Existence of the Light Resonance and its Implications to Hadron Physics", Kyoto, Japan, 11-14 June 2000, KEK-Proceedings 2000-4, Dec. 2000, 224-231 (hep-ph/0008135).

[12] E.M. Aitala et al. (E791 collaboration), Phys. Rev. Lett. 86, 770 (2001).

[13] E.V. Veliev, J. Phys. G: Nucl. Part. Phys., 35 , 035004 (2008).

[14] A. Gokalp, O. Yilmaz, Phys. Rev. D 64, 034012 (2001).

[15] M. Bellac, Thermal Field Theory, Cambridge U.P. Cambridge (1996).

[16] A. Das, Finite Temperature Field Theory, World Scientific (1999).

[17] K. Saito, K. Tsushima, A.W. Thomas, A.G. Williams, Phys.Lett. B 433, 243 (1998).

[18] D. Blaschke, G. Burau, M. K. Volkov and V. L. Yudichev, Eur. Phys. J. A 11, 319 (2001).

[19] J. Gasser and H. Leutwyler, Phys. Lett. B 184, 83 (1987).

[20] Gerber and H. Leutwyler, Nucl. Phys. B 231, 387 (1989).

[21] D.E. Miller, Acta Phys. Pol. B 28, 2937 (1997), arXiv:hep-ph/0008031

[22] H. Leutwyler, in QCD 20 Years Later Vol. 2, World Scientific, Singapore, 693 (1993).

[23] C.A. Dominguez, M. Loewe, J.C. Rojas, JHEP 08, 040 (2008). 